\title{
Animal Models of MS Reveal Multiple Roles of Microglia in Disease Pathogenesis
}

\author{
Zhen Gao and Stella E. Tsirka \\ Program in Neurosciences, Department of Pharmacological Sciences, Stony Brook University, Stony Brook, \\ NY 11794-8651, USA \\ Correspondence should be addressed to Stella E. Tsirka, stella@pharm.stonybrook.edu
}

Received 1 June 2011; Revised 5 September 2011; Accepted 5 September 2011

Academic Editor: Mamede de Carvalho

Copyright (๑) 2011 Z. Gao and S. E. Tsirka. This is an open access article distributed under the Creative Commons Attribution License, which permits unrestricted use, distribution, and reproduction in any medium, provided the original work is properly cited.

\begin{abstract}
Multiple sclerosis (MS) is a progressive inflammatory and demyelinating disease that affects more than 2.5 million people worldwide every year. Current therapies use mostly disease-modifying drugs, focusing on blocking and regulating systemic functions and the central nervous system (CNS) infiltration of immune cells; however, these therapies only attenuate or delay MS symptoms, but are not effective in halting the disease progression. More recent evidence indicated that regulation of inflammation within the CNS might be a better way to approach the treatment of the disease and microglia, the resident immune cells, may be a promising target of therapeutic studies. Microglia activation classically accompanies MS development, and regulation of microglia function changes the outcome of the disease. In this paper, we review the contributions of microglia to MS pathogenesis and discuss microglial functions in antigen presentation, cytokine release, and phagocytosis. We describe data both from animal and human studies. The significant impact of the timing, intensity, and differentiation fate of activated microglia is discussed, as they can modulate MS outcomes and potentially be critically modified for future therapeutic studies.
\end{abstract}

\section{Background}

Multiple sclerosis (MS) is a progressive autoimmune inflammatory and demyelinating disease of the central nervous system (CNS). The pathological hallmarks of MS are white matter demyelination, inflammation, axon damage, and blood-brain barrier (BBB) disruption [1-3]. The etiology of MS is still not clear, but MS is classically characterized by proinflammatory $\mathrm{T}$ helper (Th) cells, Th1 and Th17 infiltration into the CNS $[1,4]$. However, a large number of studies suggested that MS may be initiated within the CNS in the absence of peripheral immune cell infiltration $[5,6]$. Axon injury has been observed and reported very early during the development of MS, independently of lymphocyte infiltration and myelin damage $[7,8]$. Oligodendrocyte (OL) apoptosis and microglia activation have been observed in MS specimens that did not yet show lymphocytic infiltration $[6,9]$. Furthermore, MS is known to be attributed to viral infections, genetic background, and environment factors [2]. Therefore, MS may be not one disease, but rather a collection of different syndromes presenting themselves with inflammation and demyelination and multiple mechanisms underlying the etiology of the disease.

Because of the complex etiology of MS, it is hard to develop a single animal model to exactly mimic the condition and symptoms of MS patients. Several animal models have been developed in different animal species to focus on different aspects of the disease. Experimental autoimmune encephalomyelitis (EAE) is the most commonly used model for MS. It is induced by immunization of mice and rats, primarily, with myelin antigens including myelin oligodendrocyte glycoprotein (MOG), myelin basic protein (MBP), and proteolipid protein (PLP) [10]. The immunization is either active (administration of the specific antigens) or passive (administration of myelin-specific T cells). Several MS features are recapitulated by EAE, including paralysis, weight loss, demyelination, and inflammation in the CNS. In the EAE model, activated myelin-specific T cells, mainly Th1 and Th17 cells, contribute to the compromise of the BBB and migrate into the CNS. In the CNS, infiltrating and local 
antigen presenting cells (APCs) present antigens to reactive $\mathrm{T}$ cells, leading to further inflammation, demyelination, and axon damage $[1,4,10]$. However, the symptoms and animal susceptibility of EAE depend on the types of immunizing antigens and strains or species of animals used. Not all myelin antigens work in every rodent strain, and different combinations may induce animal models reflecting different subtypes and clinical course of $\mathrm{MS}$. $\mathrm{H}-2^{\mathrm{U}}$ mice, especially the PL/J strains, are highly susceptible to MBP-induced EAE. SJL mice are susceptible to PLP-induced EAE and result in a remitting-relapsing EAE course. However, the most commonly used strain is the C57BL/6(B6) mice, as many transgenic and knockout animals are maintained on this genetic background. The C57BL/6 mice are not susceptible to MBP-induced EAE; they develop symptoms only after immunization with MOG. They can exhibit either mild/ transient or severe/chronic phages depending on dosage of myelin components and immunization times [11]. For rat EAE, the most commonly used strain is the Lewis rats that are sensitive to both MBP and MOG-induced disease.

Theiler's murine encephalomyelitis virus-induced demyelinating disease (TMEV-IDD) is another inflammatory model of MS. Different from EAE, TMEV-IDD is initiated by virus infection. Responses from immune cells in this model, such as the cell types that react and the timing of reaction, may differ from $\mathrm{EAE}[12,13]$. Along similar lines, subcutaneous injection of BCG (bacillus Calmette-Guérin) has been shown to induce a delayed-type hypersensitivity (DTH) response accompanied by infiltration of macrophages and lymphocytes, the breakdown of the blood-brain barrier and immunoreactive myelin loss [14].

There are other models that induce chemical injury, such as cuprizone, lysolecithin and ethidium bromide, and result in focal demyelination in the white matter. These models are valued for studying the mechanism of demyelination/ remyelination (as remyelination is initiated upon termination of the chemical injury reagent), but they cannot account for the whole picture of MS, because they are devoid of a massive leukocyte infiltration $[13,15,16]$. Compared to these models, EAE, even though it has the drawback that the disease onset is specifically due to a defined antigen presentation as opposed to MS, appears to relatively better reflect and recapitulate critical features and progressions of MS; therefore, most of the current mechanism studies and treatments for MS still mainly rely on EAE models [17].

Current therapies focus on blocking/regulating functions and CNS infiltration of peripheral immune cells. Application of interferon- $\beta$ (IFN- $\beta$ ), glucocorticoids (GCs), and glatiramer acetate have been shown to be effective in slowing or delaying disease progression. However, no therapy has been shown to stop long-term progression, or to cure the disease $[18,19]$. One of the drawbacks is that these therapies induce a systemic inhibition of inflammation, which may block beneficial impacts of inflammation and impair recovery. Moreover, with nonspecific inhibition, the risk of developing other adverse events, such as cancer, is increased $[20,21]$. Most importantly, it is still not clear what the real initiator of the disease is, inflammation in the periphery, or damage in the CNS. Inhibiting or regulating inflammation may superficially alleviate part of symptoms, yet does not really solve the problem.

Therefore, it may be wiser to target the local immunomodulation through enhancing the beneficial aspects of inflammation within the CNS, and at the same time promote recovery by triggering and stimulating endogenous neural cells including halting death of OLs and neurons and inducing oligogenesis by neural stem cells (NSCs). However, for this goal to be accomplished, a comprehensive understanding of the interaction between CNS and periphery immune system is required, as well as understanding the interaction between neural cells and immune cells, which still constitutes the main obstacle in MS therapeutic studies due to the complexity of the neuronal network. Nevertheless, increasing evidence supports that the resident immune cells, microglia, functioning as a bridge between the CNS and immune systems, are critical for MS pathogenesis. Regulation of microglial activation may become a new promising target for therapy [22-25]. In this review, we discuss functions of microglia in MS, EAE and other experimental studies and review evidence implicating the critical roles of microglia in the complicated neural-immune networks.

\section{Microglia in MS}

Microglia derive from myeloid origin and acquire properties including cytokine production, phagocytosis, and antigen presentation, which allow microglia to play critical roles in both innate and adaptive immunity $[23,26]$. Resting microglia have a ramified morphology and can actively survey their surroundings sending out long processes to sense events occurring in their microenvironment [23]. Upon activation, microglia alter their morphology from a ramified state to assume an amoeboid shape [27]. They change expression levels of cytokines, chemokines, and surface molecules produced to respond to injury in the CNS [23]. It has been shown that microglia activation is an early event in different disease models $[9,23,28]$. Depending on their unique functions different from other resident cells in the CNS, microglia inevitably play an essential role in MS.

2.1. Microglia Are Required for Pathogenesis. Microglial activation was observed in both active demyelinating lesions and inflammatory nondemyelinating areas of MS brain and it persisted for the whole course of the disease [29, 30]. Activated microglia were found to be attached to half-damaged myelin sheaths [18]. Activated microglia in the active demyelinating areas were immunopositive for intracellular MBP [31]. Marik et al. showed that microglia became activated before demyelination was visualized and measurable [32]. This finding was consistent with studies using PET imaging showing that microglia activation was evident in the normal appearing white matter (NAWM), the tissue area devoid of leukocyte infiltration, demyelination, or BBB disruption [6]. Taken together, microglia activation during MS may be involved in the development and expansion of the disease. To clarify the roles of microglia activation in the pathogenesis of MS, Heppner et al. induced EAE to transgenic 
CD11b-HSVTK mice, in which herpes simplex virus thymidine kinase expression was driven by the monocyte/macrophage/microglia CD11b promoter, with the intention to deplete microglia activation during the disease. When these CD11b-HSVTK animals are treated with ganciclovir (GCV), all the actively proliferating cells that express the transgene (macrophages, monocytes, and microglia) die. Microglia "paralysis" (as described by the authors), mediated by ganciclovir (GCV) application, resulted in attenuation of disease severity, inflammation, and demyelination [24]. Other studies using either the macrophage/microglia inhibitory factor MIF (the tripeptide TKP) or minocycline to inhibit microglia activation also showed ameliorated EAE symptoms $[22,33,34]$. Therefore, microglia activation is a necessary component in MS pathogenesis, and inhibition of microglia activation appears to be beneficial for disease progression.

However, these results do not necessarily mean that activated microglia only contribute adversely to the disease. Studies showed that the functions of activated microglia in MS were complex and could lead to both beneficial and detrimental outcomes depending on the form and the timing of activation [22, 29, 34, 35]. Here, microglial functions in MS will be discussed in detail examining three aspects: antigen presentation, cytokine release, and phagocytosis.

2.2. Antigen Presentation. During MS and EAE, T helper (Th) cells are key mediators of the disease. In MS, Th1 and Th17 are shown to be the main pathogenic $\mathrm{T}$ cells as they promote $\mathrm{BBB}$ disruption, demyelination, and neurodegeneration [36, 37]. Anti-inflammatory Th2 and regulatory T cells (Treg) normally protect against autoimmunity by inducing tolerance of self-antigens, but their functions are impaired during early disease [4].

Antigen presentation is a critical process for $\mathrm{T}$ cell activation and modulation of their function. Antigens presented via the major histocompatibility complex (MHC-class I and MHC-class II) on the APCs are required to initiate CD8 and CD4 T cell activation. The co-stimulatory signals between CD80, CD86, and CD40 on APCs and CD28, CTLA4 , and CD40L on $\mathrm{T}$ cells are essential for full $\mathrm{T}$ cell activation. Without such costimulatory signaling, MHC-TCR ( $\mathrm{T}$ cell receptor) binding can lead to $\mathrm{T}$ cell death $[1,4$, 13, 38]. Dendritic cells (DCs), the classically established APCs, take the responsibility to initiate/prime naïve $\mathrm{T}$ cell activation [13, 39-42]. It has been reported that microglia only acquire the ability of antigen presentation upon activation, and that activated microglia favor the reactivation of primed $\mathrm{T}$ cells and regulate their differentiation $[36,40$, 41].

In the MS brain, activated microglia were shown to accumulate in all plaque regions express MHC-class II molecules and to be MBP-positive [31, 43, 44], suggesting that activated microglia in MS may also regulate $\mathrm{T}$ cell functions and lesion formation. Studies using culture systems and animal models further confirmed this observation and elucidated possible mechanisms. In vitro experiments showed that microglia gave rise to DCs after stimulation by the growth factor Granulocyte-macrophage colony-stimulating factor (GM-CSF) [41]. Coculture of microglia with IFN $\gamma^{+}$ Th1 cells and IFN $\gamma^{+}$IL- $17^{+}$Th1/Th17 cells increased the expression of MHC class II, CD40, CD80, and CD86 on microglia. Moreover, the presence of microglia resulted in significant increase of IL- $1 \beta$, TNF- $\alpha$, and IL- 6 expression in Th1/Th17 cultures, which further promoted the differentiation of proinflammatory Th cells. However, microglia/Th1 cocultures did not significantly affect cytokine release, suggesting that microglia may have different regulatory effects on Th1 and Th17 cells [36].

In vivo, upregulation of MHC class I and II, CD40, CD80, and CD86 expression on activated microglia was observed $[36,38,45,46]$. During EAE, the majority of activated microglia express higher levels of MHC molecules at all stages (initiation, peak, and recovery), and their expression correlates with disease progression and $\mathrm{T}$ cell infiltration $[36,47]$. It is well accepted that there is an upregulation of the co-stimulatory molecules CD80, CD86, and CD40 on the microglial surface, but the timing and intensity of this increased expression depend on the type of stimulation and microglial markers used. Both CD80 and CD86 are expressed on the surface of $\mathrm{MHCII}^{+} \mathrm{F} 4 / 80^{+}$microglia/ macrophages in TMEV virus infected mice [45]. Murphy et al. showed in an EAE model that increased expression of co-stimulatory molecules on $\mathrm{CD} 11^{+} \mathrm{CD} 45^{\text {high }}$ activated microglia/macrophages correlated with EAE progression [36]. However, Almonlda et al. showed that only a subset of tomato lectin ${ }^{+}$microglia/macrophages expressed CD86 at all stages, whereas, Iba- $1^{+}$microglia expressed CD86 only at the recovery stage of EAE, while there was no CD80 expression at any time point [47]. Due to lack of co-stimulatory signaling on some cells, it is possible that activated microglia may induce apoptosis of $\mathrm{T}$ cells.

In addition to the classically activating co-stimulatory pathways, the inhibitory signaling of B7 homolog 1 (B7$\mathrm{H1}$ )/programmed death receptor-1 (PD-1) between microglia and $\mathrm{T}$ cells is another key regulator of $\mathrm{T}$ cell functions $[13,46,48]$. In acute MS lesion areas, activated microglia were B7-H1 positive [49]. Moreover, B7-H1 expression in microglia was higher than that in astrocytes and splenocytes [50]. In animal models, $\mathrm{B} 7-\mathrm{H1}^{-/-}$mice or mice receiving anti-B7-H1 treatment showed worse disease symptoms, accompanied by increased demyelination and inflammation in the CNS $[46,51]$.

Although they present antigens to $\mathrm{T}$ cells, activated microglia do not function as mature DCs (mDCs): a high microglia/T cell ratio was required to induce proliferation of naïve T cells [52]. Activated microglia expressed only low levels of co-stimulatory molecules compared with mDCs during EAE [38, 47]. It has also been shown that activated microglia do not express fascin, a marker for DC maturation, suggesting that microglia may function as immature DCs to induce tolerance of $\mathrm{T}$ cells to antigens $[47,53$, 54]. Collectively, microglia present antigen to $T$ cells and regulate $\mathrm{T}$ cell functions throughout the whole process of MS disease. The interaction between microglia and $\mathrm{T}$ cells does not just promote $\mathrm{T}$ cell activation and differentiation, but also regulates inhibition through presenting inhibitory signaling. 
2.3. Cytokine and Other Mediators. Cytokine release from activated microglia has been extensively studied both in vivo and in vitro using different types of stimulation [5557]. TMEV infection, lipopolysaccharide (LPS), and IFN- $\gamma$ induce microglial expression of proinflammatory cytokines and mediators, such as nitric oxide (NO) and TNF- $\alpha$, to promote inflammation and antigen presentation. These "classically activated" microglia are defined as M1 microglia. The majority of activated microglia early in MS and animal models were M1 microglia. On the other hand, IL-4 and IL13 promote "alternatively activated" microglia (M2) differentiation. M2 microglia produce anti-inflammatory mediators including IL-4 and IL-10 to induce tissue repair [23, 28, 56]. M2 microglia (CD163+) were observed throughout the acute active lesions and the hypercellular rim of chronic active lesion in MS patients [31]. Studies from EAE and other models indicated that M1 and M2 microglia may have distinct functions in MS $[29,58]$.

2.3.1. Microglia and Neurons. Glutamate excitotoxicity-induced neurodegeneration has been described in MS and observed in the EAE model [5, 8]. EAE animals showed abnormal synaptic transmission with increased spontanous excitatory postsynaptic potential (sEPSP) frequency and a slower decay phase. Application of TNF- $\alpha$ or M1 microglia to brain slice cultures reproduced these defects. However, M1 microglia treated with anti-TNF receptor (TNFR) abolished synaptic defects suggesting that microglia regulate synaptic transmission via TNF- $\alpha$ signaling [5]. Furthermore, medium from M1 microglia significantly decreased cortical neuronal survival, whereas M2-derived medium did not have any effect [28]. Dorsal root ganglia (DRG) neurons cultured with M1 medium extended short and highly branched neurites, but those cultured with M2 medium showed bipolar morphology with longer processes. In addition, M2 microglia, together with Chondroitinase $\mathrm{ABC}$ (ChABC), significantly increased axon growth on inhibitory substrates of Chondroitin sulfate proteoglycan (CSPG) compared with M1 [28].

2.3.2. Microglia and Oligodendrocyte/Oligogenesis. The mechanisms by which activated microglia promote demyelination and impair remyelination possibly include both induction of OL death and attenuation of oligogenesis. It has been reported that activated microglia phagocytosed myelin debris in MS and EAE [58]. Activated microglia in MS were MBP-positive, indicative of microglial phagocytosis of oligodendrocyte components; in addition, medium from M1 microglia significantly decreased survival rate of OLs in culture $[31,59]$.

It is not entirely clear how microglia affect oligogenesis per se, but evidence has indicated that microglia activation impaired the function of both neural stem cells (NSCs; indirect effect on oligodendrocyte precursor cells, OPCs, since OPCs derive from NSCs), and OPCs (direct effect). Enlargement of subventricular zone (SVZ) and increased proliferation of endogenous NSCs were observed during EAE and local demyelination models $[34,60]$. However, temporal studies indicated that responses of NSCs were transient at the acute stage and completely lost in the chronic stages [34]. Moreover, increased NG2 ${ }^{+}$OPCs clustered in the SVZ but did not migrate into the white matter [60]. Experimental evidence showed that the proinflammatory microenvironment by M1 microglia contributed to the insufficient repair. Activated microglia were observed in the NSC niche in contact with the NSCs [60]. Medium from M1 microglia decreased the number of OPCs and mature OLs differentiated from NSCs. TNF- $\alpha$ inhibition dramatically downregulated this effect, suggesting that TNF- $\alpha$ primarily mediated the detrimental effect of M1 microglia [35, 59, 61]. Inhibition of microglia by minocycline significantly increased proliferation and differentiation of OPCs in EAE [34]. Therefore, although endogenous NSCs/OPCs do respond during the disease, their reactivity is not sufficient to overcome inhibition from M1 microglia or to promote efficient oligogenesis.

Due to the deficits of endogenous NSC/OPCs functions in MS, transplantation of NSCs has been adopted to promote recovery. Several studies showed that NSC transplantation dramatically attenuated disease severity, promoted remyelination, and inhibited inflammation [62-64]. Like endogenous NSCs, transplanted NSCs also expressed receptors for cytokine and chemokines; therefore, their migration and functions were under regulation by the inflammatory environment $[65,66]$. The presence of an anti-inflammatory environment, induced by IL-10, significantly enhanced adult NSC-induced functional recovery from EAE [65]. However, transplanted NSCs can also regulate immune cell functions in a dose-dependent manner $[63,67,68]$. In vitro cultured neurospheres significantly inhibited proliferation of MOGspecific lymph node cells and switched their cytokine profile from Th1 to Th2 [63]. Interestingly, there is no evidence supporting the notion that transplanted NSCs may have functions that are not present in the endogenous NSCs, suggesting that numbers or intensity of NSCs during the disease might be a critical factor determining the outcomes. Even though a proinflammatory environment still inhibits functions of transplanted NSCs, because a large number of NSC cells are transplanted, a percentage of these transplanted NSCs can still survive after "dealing with" and surviving the attacks of inflammatory cells. It would then be these remaining NSCs that migrate and function to promote recovery. If this is the case, then the hypothesis that promoting a balance or homeostasis between CNS responses and immune insults is fundamental for disease recovery.

2.3.3. Microglia and $T$ Cells. In addition to the regulation of $\mathrm{T}$ cell activation via antigen presentation (discussed in Section 2.2), activated microglia could direct $\mathrm{T}$ cell migration and differentiation through modulation of the chemokine/ cytokine environment.

Chemokines released from activated microglia contribute to leukocytes migration/infiltration into the CNS. In vitro, TMEV and IL-17A stimulated microglia expressed CCL2, CXCL3, and CCL12, which had been shown to promote the migration/infiltration of chemokine receptorexpressing leukocytes into the brain [69-71]. In MS plaques 
TABLE 1: Functions and outcomes of microglial activation during EAE/MS.

\begin{tabular}{|c|c|c|c|c|c|}
\hline Function & & iator & Timing of expression & Expression level & Outcomes \\
\hline \multirow[t]{3}{*}{$\begin{array}{l}\text { Antigen } \\
\text { Presentation }\end{array}$} & \multirow[t]{2}{*}{ Activating } & $\begin{array}{l}\text { MHC class I, MHC } \\
\text { class II }\end{array}$ & $\begin{array}{l}\text { All stages (initiation, } \\
\text { peak and recovery) }\end{array}$ & $\begin{array}{l}\text { Significantly } \\
\text { increased }\end{array}$ & $\begin{array}{l}\text { Present antigen, initiate } \\
\mathrm{T} \text { cell activation; Induce } \\
\text { apoptosis without } \\
\text { co-stimulatory } \\
\text { molecules }\end{array}$ \\
\hline & & CD80, CD86, CD40, & $\begin{array}{l}\text { Depends on cell } \\
\text { markers chosen }\end{array}$ & $\begin{array}{l}\text { Increased, but lower } \\
\text { than DCs }\end{array}$ & $\begin{array}{l}\text { Fully activate T cell with } \\
\text { MHC molecules }\end{array}$ \\
\hline & Inhibitory & B1-H7 & Not examined & Increased & $\begin{array}{l}\text { T cell activation } \\
\text { inhibition }\end{array}$ \\
\hline \multirow{3}{*}{$\begin{array}{l}\text { Cytokine/ } \\
\text { chemokine release }\end{array}$} & $\begin{array}{l}\text { Proinflammatory } \\
\text { (M1) }\end{array}$ & $\begin{array}{l}\text { IL- } 1 \beta, \text { TNF- } \alpha, \text { IL-6, } \\
\text { NO, IFN- } \gamma, \text { IL-12 }\end{array}$ & $\begin{array}{l}\text { High at onset, } \\
\text { sustained at all stages } \\
\text { (initiation, peak and } \\
\text { recovery) }\end{array}$ & $\begin{array}{l}\text { Dramatically } \\
\text { increased, dominant }\end{array}$ & $\begin{array}{l}\text { Induce synaptic deficits; } \\
\text { death of neurons and } \\
\text { OLs; } \\
\text { Induce Th1 and Th17 } \\
\text { differentiation; } \\
\text { Induce disfunction of } \\
\text { NSCs/OPCs }\end{array}$ \\
\hline & $\begin{array}{l}\text { Anti-inflammatory } \\
\text { (M2) }\end{array}$ & IL- 4, IL-10, TGF- $\beta$ & $\begin{array}{l}\text { Low at onset, } \\
\text { increased at later time } \\
\text { points }\end{array}$ & $\begin{array}{l}\text { Increased, lower than } \\
\text { M1 }\end{array}$ & $\begin{array}{l}\text { Promote growth of long } \\
\text { dendrites; } \\
\text { Induce Th2 and Treg } \\
\text { differentiation; } \\
\text { Increase proliferation } \\
\text { and differentiation of } \\
\text { NSCs/OPCs }\end{array}$ \\
\hline & Chemokines & $\begin{array}{c}\text { CCL2, CXCL3, } \\
\text { CCL12, CCL4, CCL5 }\end{array}$ & Varies & Increased & $\begin{array}{l}\text { Regulate migration of T } \\
\text { cells, NSCs and OPCs }\end{array}$ \\
\hline \multirow{2}{*}{ Phagocytosis } & Activating & $\begin{array}{c}\text { CR3, SRA, Fc } \gamma \text { R, } \\
\text { TREM } 2\end{array}$ & Not examined & Increased & $\begin{array}{l}\text { Facilitate recognition of } \\
\text { myelin, remove myelin } \\
\text { debris }\end{array}$ \\
\hline & Inhibitory & $\operatorname{SIRP} \alpha$ & Not examined & Decreased & $\begin{array}{l}\text { Downregulate } \\
\text { phagocytosis, induce } \\
\text { self-tolerance }\end{array}$ \\
\hline
\end{tabular}

and TMEV-IDD, increased expression of CCL2, CCL4, and CCL5 was observed in activated microglia [72-75]. Inhibition of microglial activation by the tripeptide MIF/TKP significantly decreased T cell numbers in the CNS [22].

Activated microglia express cytokines required for $\mathrm{T}$ cell differentiation. M1 microglia stimulated by TMEV and LPS produced high levels of TNF- $\alpha$, IL-6, and IL-12, which promoted the differentiation and expansion of Th1 and/or Th17 cells $[13,39]$. Coculture of microglia and Th1/Th17 cells increased IL- $1 \beta$ and IL- 6 release, which were responsible for further expansion of Th1/Th17 cells [36]. M2 microglia expressing IL-4 and IL-10 were required for Th2 and Treg differentiation [4]. TGF- $\beta$ released from M2 directly promoted Treg differentiation and could regulate Th17 functions indirectly through inhibition of Th1 and Th2 differentiation $[4,76]$. To conclude, M1 microglia promote $\mathrm{T}$ cell differentiation toward Th1 and Th17 fates and synergistically induce demyelination and neurodegeneration. On the other hand, M2 microglia, together with anti-inflammatory T cells (Th2 and Treg), protect the system from damage and promote recovery.
2.3.4. Timing and Intensity of Microglia Activation. Both M1 proinflammatory microglia and M2 anti-inflammatory microglia contribute to MS progression, but M1 microglia are dominant early and during the whole process. This M1 prevalence is induced by differences in timing and intensity of M1 and M2 microglial activation. M1 microglia are observed in the CNS since the onset of the EAE and are sustained through the whole process. However, a delayed M2 differentiation has been described, accompanied by persisting low numbers of M2 microglia during EAE, which resulted in an imbalance between the two microglial subtypes and high M1/M2 ratio $[29,36]$. Because of this delayed and lower M2 differentiation, even though the M2 population has a protective effect, it still cannot overcome the detrimental effects of the M1 population. To promote recovery through regulating microglia functions, changing the M1/M2 ratio would be a key point. In line with this, injection of IL10/IL-13 activated M2 macrophages/monocytes significantly inhibited microglial activation and suppressed EAE symptoms presumably through rebalancing the M1/M2 ratio [29]. Similarly, the beneficial effects of minocycline on EAE 
symptoms might be due to its biased inhibition of M1 microglia $[34,59,77]$.

Nonetheless, balancing the M1/M2 ratio does not mean that total suppression of the M1 population is good for the disease, since M1 microglia are not always bad. Butovsky et al. showed that moderate M1 microglia activation induced by IFN- $\gamma$ could increase neurogenesis and oligogenesis, whereas excessive activation inhibited these effects [35, 61]. It is also reported that controlled microglial activation and $\mathrm{T}$ cell infiltration promoted recovery from optic nerve injury [78]. Therefore, M1 microglia could be beneficial for tissue recovery if the extent of their activation is controlled within a well-defined range.

2.3.5. Phagocytosis. Phagocytosis of myelin debris by activated microglia was observed in MS lesions and it was an essential response to promote regeneration $[25,58]$. Phagocytic ability of microglia is mediated by interactions between myelin ligands and receptors on microglia. Myelin stimulated microglial activation through the binding of complement receptor 3 (CR3), scavenger receptor $\mathrm{AI} / \mathrm{II}$ (SRA), and $\mathrm{Fc} \gamma$ receptor $(\mathrm{F} c \gamma \mathrm{R})$, which then induced further activation and phagocytosis [79-81]. On the other hand, signaling pathways like myelin-CD47 binding to signal regulatory protein- $\alpha$ $(\operatorname{SIRP} \alpha)$ can down regulate microglial phagocytosis [82]. Such inhibitory pathways were originally thought to induce self-tolerance by protecting healthy cells from attacks by phagocytes. However, the expression of CD47 was reported to be significantly decreased in MS lesions, suggesting the onset of uncontrolled phagocytosis by microglia [19]. Therefore, although phagocytosis of myelin debris is beneficial for recovery, its impact on disease progression is still under debate.

The inflammatory environment is a key regulator for microglia phagocytic function. Application of proinflammatory cytokines reduced phagocytosis by macrophages/microglia [83]. Activated microglia dramatically increased expression of the microglial triggering receptor expressed on myeloid cells 2 (TREM2) during EAE [84, 85]. Studies showed that TREM2 stimulated phagocytosis in vitro and promoted an anti-inflammatory state in EAE $[84,86]$. Inhibition of TREM2 resulted in exacerbated EAE symptoms [87]. Furthermore, pathways mediating microglial phagocytosis, as mentioned above, including CR3/MAC-1 and CD47/SIRP $\alpha$, were not restricted to regulating phagocytosis, but also had broad effects on microglial activation and migration [18]. It is suggested then that phagocytic function of microglia is tightly correlated with and under control of the status of microglial activation in MS. Insufficient myelin clean-up may be due to blockade of phagocytosis by an unbalanced $\mathrm{M} 1 / \mathrm{M} 2$ ratio.

\section{Conclusions}

Microglial activation is not just a hallmark of MS, but is required for disease pathogenesis. Activated microglia differentiate into M1 and M2 microglia and contribute to both protective and detrimental aspects of the inflammatory process through antigen presentation, cytokine release, and phagocytosis (Table 1). Regulating microglial functions could both affect the level of inflammatory insults and change local responses from neural cells. However, beneficial effects will only be brought when microglial activation is well-defined in time, intensity, and direction of differentiation, which are still unclear. Further studies are required to clarify and precisely determine these parameters to fully understand the functions of microglia in MS.

\section{Acknowledgments}

The authors would like to thank Dr Michael Frohman and members of the Tsirka lab for paper editing and helpful discussions. This work was supported by NIH R0142168 (SET) and a NMSS Center Grant.

\section{References}

[1] S. Dhib-Jalbut, "Pathogenesis of myelin/oligodendrocyte damage in multiple sclerosis," Neurology, vol. 68, no. 22, supplement 3, pp. S13-S54, 2007.

[2] T. Holmoy and A. L. Hestvik, "Multiple sclerosis: immunopathogenesis and controversies in defining the cause," Current Opinion in Infectious Diseases, vol. 21, no. 3, pp. 271-278, 2008.

[3] R. P. Lisak, "Neurodegeneration in multiple sclerosis: defining the problem," Neurology, vol. 68, no. 22, supplement 3, pp. S5S12, 2007, discussion S43-S54.

[4] J. M. Fletcher, S. J. Lalor, C. M. Sweeney, N. Tubridy, and K. H. G. Mills, "T cells in multiple sclerosis and experimental autoimmune encephalomyelitis," Clinical and Experimental Immunology, vol. 162, no. 1, pp. 1-11, 2010.

[5] D. Centonze, L. Muzio, S. Rossi et al., "Inflammation triggers synaptic alteration and degeneration in experimental autoimmune encephalomyelitis," Journal of Neuroscience, vol. 29, no. 11, pp. 3442-3452, 2009.

[6] J. M. van Noort, P. J. van den Elsen, J. van Horssen, J. J. Geurts, P. van der Valk, and S. Amor, "Preactive multiple sclerosis lesions offer novel clues for neuroprotective therapeutic strategies," CNS \& Neurological Disorders Drug Targets, vol. 10, no. 1, pp. 68-81, 2011.

[7] P. M. Matthews, E. Pioro, S. Narayanan et al., "Assessment of lesion pathology in multiple sclerosis using quantitative MRI morphometry and magnetic resonance spectroscopy," Brain, vol. 119, no. 3, pp. 715-722, 1996.

[8] D. Pitt, P. Werner, and C. S. Raine, "Glutamate excitotoxicity in a model of multiple sclerosis," Nature Medicine, vol. 6, no. 1, pp. 67-70, 2000.

[9] M. H. Barnett and J. W. Prineas, "Relapsing and remitting multiple sclerosis: pathology of the newly forming lesion," Annals of Neurology, vol. 55, no. 4, pp. 458-468, 2004.

[10] B. Hemmer, J. J. Archelos, and H. P. Hartung, "New concepts in the immunopathogenesis of multiple sclerosis," Nature Reviews Neuroscience, vol. 3, no. 4, pp. 291-301, 2002.

[11] S. Kuerten and D. N. Angelov, "Comparing the CNS morphology and immunobiology of different EAE models in C57BL/6 mice-a step towards understanding the complexity of multiple sclerosis," Annals of Anatomy, vol. 190, no. 1, pp. $1-15,2008$. 
[12] A. Denic, A. J. Johnson, A. J. Bieber, A. E. Warrington, M. Rodriguez, and I. Pirko, "The relevance of animal models in multiple sclerosis research," Pathophysiology, vol. 18, no. 1, pp. 21-29, 2011.

[13] E. M. Chastain, D. S. Duncan, J. M. Rodgers, and S. D. Miller, "The role of antigen presenting cells in multiple sclerosis," Biochimica et Biophysica Acta, vol. 1812, no. 2, pp. 265-274, 2011.

[14] M. K. Matyszak and V. H. Perry, "Delayed-type hypersensitivity lesions in the central nervous system are prevented by inhibitors of matrix metalloproteinases," Journal of Neuroimmunology, vol. 69, no. 1-2, pp. 141-149, 1996.

[15] O. Torkildsen, L. A. Brunborg, K.-M. Myhr, and L. Bo, “The cuprizone model for demyelination," Acta Neurologica Scandinavica, vol. 117, supplement 188, pp. 72-76, 2008.

[16] W. F. Blakemore and R. J. M. Franklin, "Remyelination in experimental models of toxin-induced demyelination," Current Topics in Microbiology and Immunology, vol. 318, pp. 193 212, 2008.

[17] L. Steinman and S. S. Zamvil, "How to successfully apply animal studies in experimental allergic encephalomyelitis to research on multiple sclerosis," Annals of Neurology, vol. 60, no. 1, pp. 12-21, 2006.

[18] N. Koning, B. M. J. Uitdehaag, I. Huitinga, and R. M. Hoek, "Restoring immune suppression in the multiple sclerosis brain," Progress in Neurobiology, vol. 89, no. 4, pp. 359-368, 2009.

[19] N. Koning, L. Bö, R. M. Hoek, and I. Huitinga, "Downregulation of macrophage inhibitory molecules in multiple sclerosis lesions," Annals of Neurology, vol. 62, no. 5, pp. 504-514, 2007.

[20] R. A. Farrell and G. Giovannoni, "Current and future role of interferon beta in the therapy of multiple sclerosis," Journal of Interferon and Cytokine Research, vol. 30, no. 10, pp. 715-726, 2010.

[21] J. S. Wolinsky, "The use of glatiramer acetate in the treatment of multiple sclerosis," Advances in Neurology, vol. 98, pp. 273292, 2006.

[22] M. Bhasin, M. Wu, and S. E. Tsirka, "Modulation of microglial/macrophage activation by macrophage inhibitory factor (TKP) or tuftsin (TKPR) attenuates the disease course of experimental autoimmune encephalomyelitis," BMC Immunology, vol. 8, article 10, 2007.

[23] U. K. Hanisch and H. Kettenmann, "Microglia: active sensor and versatile effector cells in the normal and pathologic brain," Nature Neuroscience, vol. 10, no. 11, pp. 1387-1394, 2007.

[24] F. L. Heppner, M. Greter, D. Marino et al., "Experimental autoimmune encephalomyelitis repressed by microglial paralysis," Nature Medicine, vol. 11, no. 2, pp. 146-152, 2005.

[25] I. Napoli and H. Neumann, "Protective effects of microglia in multiple sclerosis," Experimental Neurology, vol. 225, no. 1, pp. 24-28, 2010.

[26] M. Prinz and A. Mildner, "Microglia in the CNS: immigrants from another world," Glia, vol. 59, no. 2, pp. 177-187, 2011.

[27] N. Stence, M. Waite, and M. E. Dailey, "Dynamics of microglial activation: a confocal time-lapse analysis in hippocampal slices," Glia, vol. 33, no. 3, pp. 256-266, 2001.

[28] K. A. Kigerl, J. C. Gensel, D. P. Ankeny, J. K. Alexander, D. J. Donnelly, and P. G. Popovich, "Identification of two distinct macrophage subsets with divergent effects causing either neurotoxicity or regeneration in the injured mouse spinal cord," Journal of Neuroscience, vol. 29, no. 43, pp. 13435 13444, 2009.
[29] J. Mikita, N. Dubourdieu-Cassagno, M. S. Deloire et al., "Altered M1/M2 activation patterns of monocytes in severe relapsing experimental rat model of multiple sclerosis. Amelioration of clinical status by $\mathrm{M} 2$ activated monocyte administration," Multiple Sclerosis, vol. 17, no. 1, pp. 2-15, 2011.

[30] M. Wu and S. E. Tsirka, "Endothelial NOS-deficient mice reveal dual roles for nitric oxide during experimental autoimmune encephalomyelitis," Glia, vol. 57, no. 11, pp. 1204-1215, 2009.

[31] Z. Zhang, Z. Y. Zhang, J. Schittenhelm, Y. Wu, R. Meyermann, and H. J. Schluesener, "Parenchymal accumulation of CD163+ macrophages/microglia in multiple sclerosis brains," Journal of Neuroimmunology, vol. 237, no. 1-2, pp. 73-79, 2011.

[32] C. Marik, P. A. Felts, J. Bauer, H. Lassmann, and K. J. Smith, "Lesion genesis in a subset of patients with multiple sclerosis: a role for innate immunity?" Brain, vol. 130, no. 11, pp. 28002815, 2007.

[33] A. Defaux, M. G. Zurich, P. Honegger, and F. Monnet-Tschudi, "Minocycline promotes remyelination in aggregating rat brain cell cultures after interferon-gamma plus lipopolysaccharideinduced demyelination," Neuroscience, vol. 187, pp. 84-92, 2011.

[34] S. Rasmussen, J. Imitola, A. Ayuso-Sacido et al., "Reversible neural stem cell niche dysfunction in a model of multiple sclerosis," Annals of Neurology, vol. 69, no. 5, pp. 878-891, 2011.

[35] O. Butovsky, G. Landa, G. Kunis et al., "Induction and blockage of oligodendrogenesis by differently activated microglia in an animal model of multiple sclerosis," Journal of Clinical Investigation, vol. 116, no. 4, pp. 905-915, 2006.

[36] A. C. Murphy, S. J. Lalor, M. A. Lynch, and K. H. G. Mills, "Infiltration of Th1 and Th17 cells and activation of microglia in the CNS during the course of experimental autoimmune encephalomyelitis," Brain, Behavior, and Immunity, vol. 24, no. 4, pp. 641-651, 2010.

[37] I. M. Stromnes, L. M. Cerretti, D. Liggitt, R. A. Harris, and J. M. Goverman, "Differential regulation of central nervous system autoimmunity by T H1 and TH17 cells," Nature Medicine, vol. 14, no. 3, pp. 337-342, 2008.

[38] B. Almolda, B. Gonzalez, and B. Castellano, "Antigen presentation in EAE: role of microglia, macrophages and dendritic cells," Frontiers in Bioscience, vol. 16, no. 3, pp. 1157-1171, 2011.

[39] B. Becher, I. Bechmann, and M. Greter, "Antigen presentation in autoimmunity and CNS inflammation: how T iymphocytes recognize the brain," Journal of Molecular Medicine, vol. 84, no. 7, pp. 532-543, 2006.

[40] R. Gandhi, A. Laroni, and H. L. Weiner, "Role of the innate immune system in the pathogenesis of multiple sclerosis," Journal of Neuroimmunology, vol. 221, no. 1-2, pp. 7-14, 2010.

[41] H. G. Fischer and G. Reichmann, "Brain dendritic cells and macrophages/microglia in central nervous system inflammation," Journal of Immunology, vol. 166, no. 4, pp. 2717-2726, 2001.

[42] A. L. Zozulya, B. D. Clarkson, S. Ortler, Z. Fabry, and H. Wiendl, "The role of dendritic cells in CNS autoimmunity," Journal of Molecular Medicine, vol. 88, no. 6, pp. 535-544, 2010.

[43] G. Raivich and R. Banati, "Brain microglia and bloodderived macrophages: molecular profiles and functional roles in multiple sclerosis and animal models of autoimmune demyelinating disease," Brain Research Reviews, vol. 46, no. 3, pp. 261-281, 2004. 
[44] E. A. Boyle and P. L. McGeer, "Cellular immune response in multiple sclerosis plaques," American Journal of Pathology, vol. 137, no. 3, pp. 575-584, 1990.

[45] J. G. Pope, C. L. Vanderlugt, S. M. Rahbe, H. L. Lipton, and S. D. Miller, "Characterization of and functional antigen presentation by central nervous system mononuclear cells from mice infected with Theiler's murine encephalomyelitis virus," Journal of Virology, vol. 72, no. 10, pp. 7762-7771, 1998.

[46] D. S. Duncan and S. D. Miller, "CNS expression of B7-H1 regulates pro-inflammatory cytokine production and alters severity of Theiler's virus-induced demyelinating disease," Plos One, vol. 6, no. 4, Article ID e18548, 2011.

[47] B. Almolda, B. Gonzalez, and B. Castellano, "Activated microglial cells acquire an immature dendritic cell phenotype and may terminate the immune response in an acute model of EAE," Journal of Neuroimmunology, vol. 223, no. 1-2, pp. 3954, 2010.

[48] C. Blank, J. Kuball, S. Voelkl et al., "Blockade of PD-L1 (B7$\mathrm{H} 1$ ) augments human tumor-specific $\mathrm{T}$ cell responses in vitro," International Journal of Cancer, vol. 119, no. 2, pp. 317327, 2006.

[49] R. De Simone, A. Giampaolo, B. Giometto et al., "The costimulatory molecule B7 is expressed on human microglia in culture and in multiple sclerosis acute lesions," Journal of Neuropathology and Experimental Neurology, vol. 54, no. 2, pp. 175-187, 1995.

[50] T. Magnus, B. Schreiner, T. Korn et al., "Microglial expression of the B7 family member B7 homolog 1 confers strong immune inhibition: implications for immune responses and autoimmunity in the CNS," Journal of Neuroscience, vol. 25, no. 10, pp. 2537-2546, 2005.

[51] S. Ortler, C. Leder, M. Mittelbronn et al., "B7-H1 restricts neuroantigen-specific $T$ cell responses and confines inflammatory CNS damage: implications for the lesion pathogenesis of multiple sclerosis," European Journal of Immunology, vol. 38, no. 6, pp. 1734-1744, 2008.

[52] E. J. McMahon, S. L. Bailey, C. V. Castenada, H. Waldner, and S. D. Miller, "Epitope spreading initiates in the CNS in two mouse models of multiple sclerosis," Nature Medicine, vol. 11, no. 3, pp. 335-339, 2005.

[53] M. V. Dhodapkar, R. M. Steinman, J. Krasovsky, C. Munz, and N. Bhardwaj, "Antigen-specific inhibition of effector T cell function in humans after injection of immature dendritic cells," Journal of Experimental Medicine, vol. 193, no. 2, pp. 233-238, 2001.

[54] M. G. Roncarolo, M. K. Levings, and C. Traversari, "Differentiation of T regulatory cells by immature dendritic cells," Journal of Experimental Medicine, vol. 193, no. 2, pp. F5-F9, 2001.

[55] T. D. Merson, M. D. Binder, and T. J. Kilpatrick, "Role of cytokines as mediators and regulators of microglial activity in inflammatory demyelination of the CNS," NeuroMolecular Medicine, vol. 12, no. 2, pp. 99-132, 2010.

[56] C. A. Colton and D. M. Wilcock, "Assessing activation states in microglia," CNS and Neurological Disorders, vol. 9, no. 2, pp. 174-191, 2010.

[57] S. Ebert, J. Gerber, S. Bader et al., "Dose-dependent activation of microglial cells by Toll-like receptor agonists alone and in combination," Journal of Neuroimmunology, vol. 159, no. 1-2, pp. 87-96, 2005.

[58] J. Bauer, T. Sminia, F. G. Wouterlood, and C. D. Dijkstra, "Phagocytic activity of macrophages and microglial cells during the course of acute and chronic relapsing experimental autoimmune encephalomyelitis," Journal of Neuroscience Research, vol. 38, no. 4, pp. 365-375, 1994.
[59] W. W. Li, A. Setzu, C. Zhao, and R. J. M. Franklin, "Minocycline-mediated inhibition of microglia activation impairs oligodendrocyte progenitor cell responses and remyelination in a non-immune model of demyelination," Journal of $\mathrm{Neu}$ roimmunology, vol. 158, no. 1-2, pp. 58-66, 2005.

[60] S. Pluchino, L. Muzio, J. Imitola et al., "Persistent inflammation alters the function of the endogenous brain stem cell compartment," Brain, vol. 131, no. 10, pp. 2564-2578, 2008.

[61] O. Butovsky, Y. Ziv, A. Schwartz et al., "Microglia activated by IL-4 or IFN- $\gamma$ differentially induce neurogenesis and oligodendrogenesis from adult stem/progenitor cells," Molecular and Cellular Neuroscience, vol. 31, no. 1, pp. 149-160, 2006.

[62] O. Einstein, D. Karussis, N. Grigoriadis et al., "Intraventricular transplantation of neural precursor cell spheres attenuates acute experimental allergic encephalomyelitis," Molecular and Cellular Neuroscience, vol. 24, no. 4, pp. 1074-1082, 2003.

[63] O. Einstein, N. Fainstein, I. Vaknin et al., "Neural precursors attenuate autoimmune encephalomyelitis by peripheral immunosuppression," Annals of Neurology, vol. 61, no. 3, pp. 209-218, 2007.

[64] S. Pluchino, A. Quattrini, E. Brambilla et al., "Injection of adult neurospheres induces recovery in a chronic model of multiple sclerosis," Nature, vol. 422, no. 6933, pp. 688-694, 2003.

[65] J. Yang, Z. Jiang, D. C. Fitzgerald et al., "Adult neural stem cells expressing IL-10 confer potent immunomodulation and remyelination in experimental autoimmune encephalitis," Journal of Clinical Investigation, vol. 119, no. 12, pp. 36783691, 2009.

[66] K. S. Carbajal, C. Schaumburg, R. Strieter, J. Kane, and T. E. Lane, "Migration of engrafted neural stem cells is mediated by CXCL12 signaling through CXCR4 in a viral model of multiple sclerosis," Proceedings of the National Academy of Sciences of the United States of America, vol. 107, no. 24, pp. 11068-11073, 2010.

[67] D. Michel-Monigadon, P. Brachet, I. Neveu, and P. Naveilhan, "Immunoregulatory properties of neural stem cells," Immunotherapy, vol. 3, supplement 4, pp. 39-41, 2011.

[68] N. Fainstein, I. Vaknin, O. Einstein et al., "Neural precursor cells inhibit multiple inflammatory signals," Molecular and Cellular Neuroscience, vol. 39, no. 3, pp. 335-341, 2008.

[69] J. Das Sarma, B. Ciric, R. Marek et al., "Functional interleukin17 receptor $\mathrm{A}$ is expressed in central nervous system glia and upregulated in experimental autoimmune encephalomyelitis," Journal of Neuroinflammation, vol. 6, article 14, 2009.

[70] J. K. Olson and S. D. Miller, "Microglia initiate central nervous system innate and adaptive immune responses through multiple TLRs," Journal of Immunology, vol. 173, no. 6, pp. 39163924, 2004.

[71] E. A. Eugenin, K. Osiecki, L. Lopez, H. Goldstein, T. M. Calderon, and J. W. Berman, "CCL2/monocyte chemoattractant protein-1 mediates enhanced transmigration of human immunodeficiency virus (HIV)-infected leukocytes across the blood-brain barrier: a potential mechanism of HIV-CNS invasion and NeuroAIDS," Journal of Neuroscience, vol. 26, no. 4, pp. 1098-1106, 2006.

[72] M. A. Lee, S. Smith, J. Palace, and P. M. Matthews, "Defining multiple sclerosis disease activity using MRI T2-weighted difference imaging," Brain, vol. 121, no. 11, pp. 2095-2102, 1998.

[73] P. S. Sorensen, B. Wanscher, C. V. Jensen et al., "Intravenous immunoglobulin $\mathrm{G}$ reduces MRI activity in relapsing multiple sclerosis," Neurology, vol. 50, no. 5, pp. 1273-1281, 1998. 
[74] J. E. Simpson, J. Newcombe, M. L. Cuzner, and M. N. Woodroofe, "Expression of monocyte chemoattractant protein-1 and other $\beta$-chemokines by resident glia and inflammatory cells in multiple sclerosis lesions," Journal of Neuroimmunology, vol. 84, no. 2, pp. 238-249, 1998.

[75] B. S. Kim, J. P. Palma, D. Kwon, and A. C. Fuller, "Innate immune response induced by Theiler's murine encephalomyelitis virus infection," Immunologic Research, vol. 31, no. 1, pp. $1-12,2005$.

[76] J. Das, G. Ren, L. Zhang et al., "Transforming growth factor $\beta$ is dispensable for the molecular orchestration of Th17 cell differentiation," Journal of Experimental Medicine, vol. 206, no. 11, pp. 2407-2416, 2009.

[77] H. Zhou, B. M. Lapointe, S. R. Clark, L. Zbytnuik, and P. Kubes, "A requirement for microglial TLR4 in leukocyte recruitment into brain in response to lipopolysaccharide," Journal of Immunology, vol. 177, no. 11, pp. 8103-8110, 2006.

[78] I. Shaked, Z. Porat, R. Gersner, J. Kipnis, and M. Schwartz, "Early activation of microglia as antigen-presenting cells correlates with $\mathrm{T}$ cell-mediated protection and repair of the injured central nervous system," Journal of Neuroimmunology, vol. 146, no. 1-2, pp. 84-93, 2004.

[79] S. Rotshenker, "Microglia and macrophage activation and the regulation of complement-receptor-3 (CR3/MAC-1)mediated myelin phagocytosis in injury and disease," Journal of Molecular Neuroscience, vol. 21, no. 1, pp. 65-72, 2003.

[80] M. E. Smith, "Phagocytic properties of microglia in vitro: implications for a role in multiple sclerosis and EAE," Microscopy Research and Technique, vol. 54, no. 2, pp. 81-94, 2001.

[81] S. Rotshenker, "The role of Galectin-3/MAC-2 in the activation of the innate-immune function of phagocytosis in microglia in injury and disease," Journal of Molecular Neuroscience, vol. 39, no. 1-2, pp. 99-103, 2009.

[82] M. Gitik, S. L. Zaltsman, P.-A. Oldenborg et al., "Myelin downregulates myelin phagocytosis by microglia and macrophages through interactions between CD47 on myelin and SIRPalpha (signal regulatory protein-alpha) on phagocytes," Journal of Neuroinflammation, vol. 8, article 24, 2011.

[83] M. E. Smith, K. Van Der Maesen, and F. P. Somera, "Macrophage and microglial responses to cytokines in vitro: phagocytic activity, proteolytic enzyme release, and free radical production," Journal of Neuroscience Research, vol. 54, no. 1, pp. 68-78, 1998.

[84] K. Takahashi, M. Prinz, M. Stagi, O. Chechneva, and H. Neumann, "TREM2-transduced myeloid precursors mediate nervous tissue debris clearance and facilitate recovery in an animal model of multiple sclerosis," Plos Medicine, vol. 4, no. 4, article e124, 2007.

[85] C. D. Schmid, L. N. Sautkulis, P. E. Danielson et al., "Heterogeneous expression of the triggering receptor expressed on myeloid cells-2 on adult murine microglia," Journal of Neurochemistry, vol. 83, no. 6, pp. 1309-1320, 2002.

[86] K. Takahashi, C. D. Rochford, and H. Neumann, "Clearance of apoptotic neurons without inflammation by microglial triggering receptor expressed on myeloid cells-2," Journal of Experimental Medicine, vol. 201, no. 4, pp. 647-657, 2005.

[87] L. Piccio, C. Buonsanti, M. Mariani et al., "Blockade of TREM2 exacerbates experimental autoimmune encephalomyelitis," European Journal of Immunology, vol. 37, no. 5, pp. 1290-1301, 2007. 


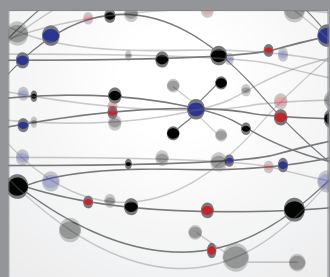

The Scientific World Journal
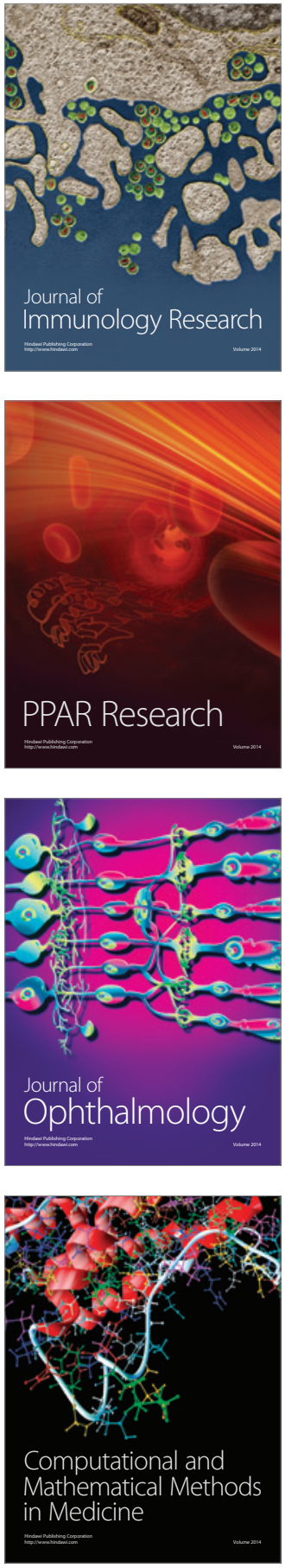

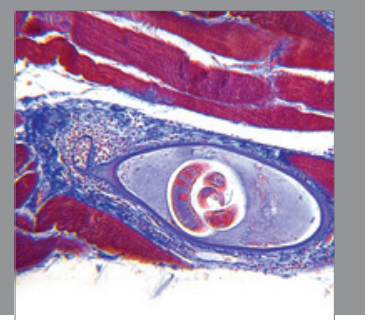

Gastroenterology

Research and Practice
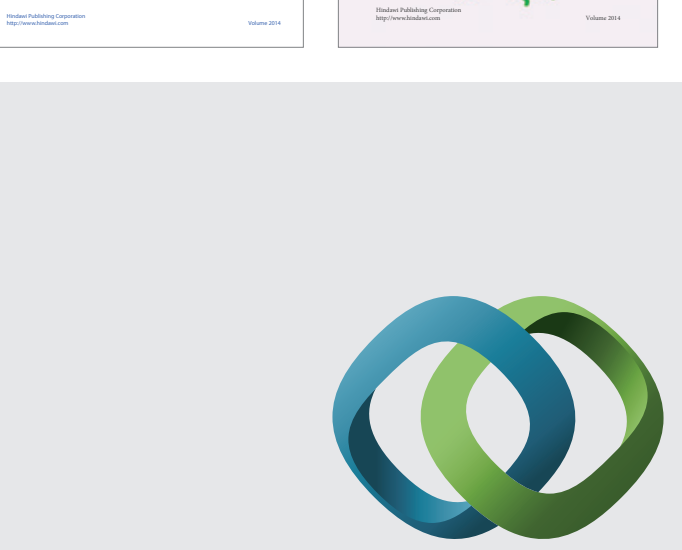

\section{Hindawi}

Submit your manuscripts at

http://www.hindawi.com
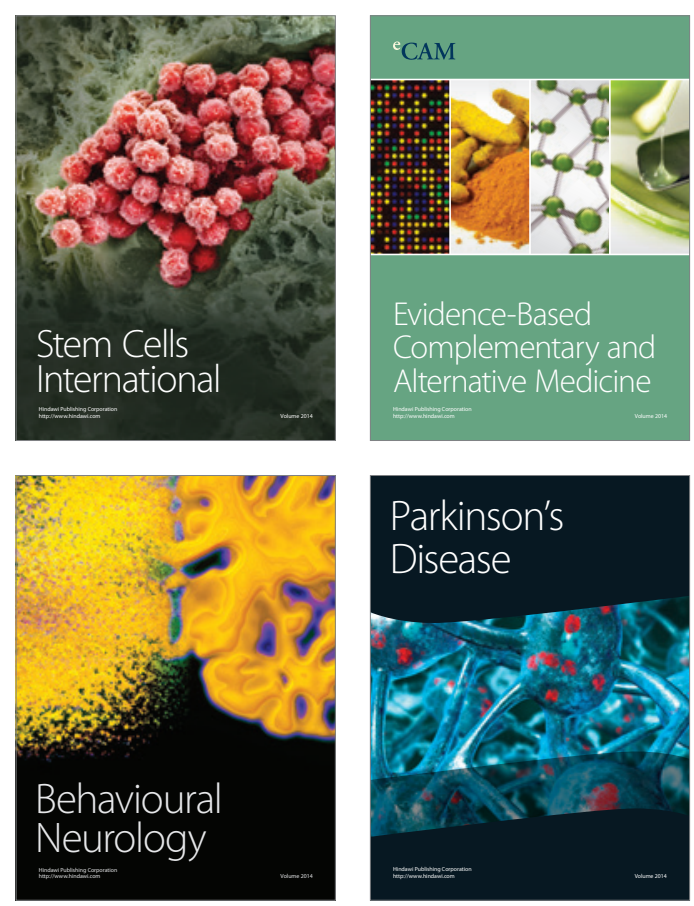

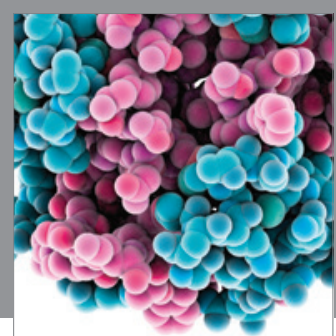

Journal of
Diabetes Research

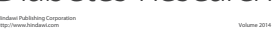

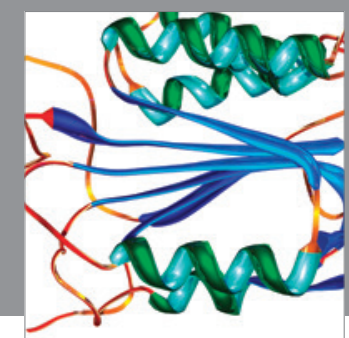

Disease Markers
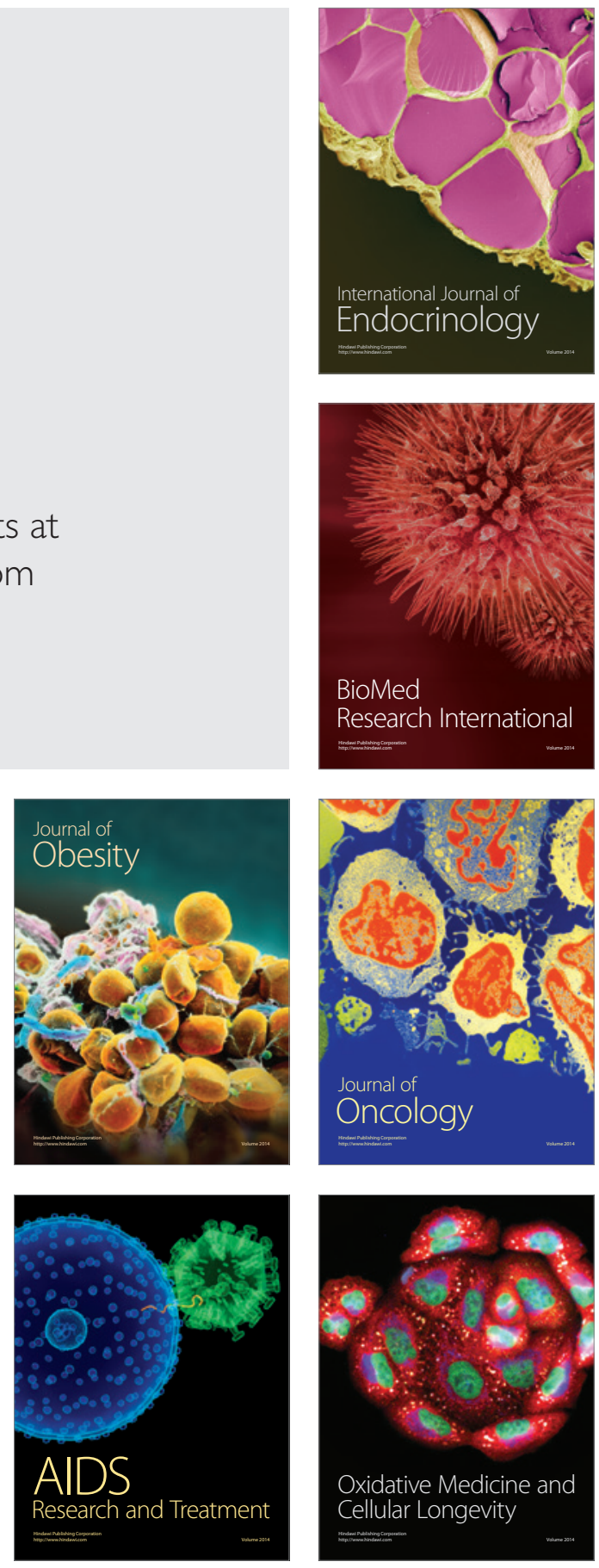